\title{
PLL Based Digital Adaptive Filter for Detecting Interharmonics
}

\author{
Ayhan Özdemir ${ }^{1}$ and Mehmet Taştan ${ }^{2}$ \\ ${ }^{1}$ Department of Electrical-Electronics Engineering, Sakarya University, Esentepe, 54187 Sakarya, Turkey \\ ${ }^{2}$ Turgutlu Vocational High School, Industrial Automation Department, Celal Bayar University, 45000 Manisa, Turkey \\ Correspondence should be addressed to Mehmet Taştan; mehmet.tastan@cbu.edu.tr
}

Received 18 March 2014; Revised 21 May 2014; Accepted 25 May 2014; Published 23 June 2014

Academic Editor: Xia Huang

Copyright ( 2014 A. Özdemir and M. Taştan. This is an open access article distributed under the Creative Commons Attribution License, which permits unrestricted use, distribution, and reproduction in any medium, provided the original work is properly cited.

Studies on the PLL (phase locked loop) based interharmonic measurements have been considerably increased recently. The method proposed in this study is a hybrid method based on the frequency analysis. The proposed method presents solutions for the leading problems about the interharmonic measurement, such as fundamental frequency shifting, spectral leakage, and picket-fence effect, using digital PLL and adaptive notch filter. In addition, a new approach is presented to solve the multizero crossing problem encountered in the analysis of the harmonic-interharmonic. Both simulative and field data tests have been performed to verify the effectiveness of the proposed method.

\section{Introduction}

In recent years, power electronics has found a broad application area in the discipline of the energy systems. Therefore, power quality problems have naturally come out. One of these problems is the interharmonics occurring in the power systems.

Interharmonic was firstly defined in the IEC-61000-2-1 (International Electrotechnical Commission) standard in 1990 and this definition was renewed with the standards of IEC-61000-2-2. Accordingly, interharmonic is any frequency which is noninteger multiple of the fundamental frequency [1]. The interharmonics are particularly generated by arc furnaces, welding machines, static frequency converters, and motor converter drivers. They cause not only some problems like overheating, shortening in the lifetime which harmonics cause, but also further problems like voltage fluctuations, subsynchronous oscillation, and flickers [2].

In the analysis of the interharmonics, the widely used leading methods are FFT (fast Fourier transformation) and DFT (discrete Fourier transformation). In these methods, the frequency resolution is limited by the windowing function. If the measurement window is not correctly synchronized with the fundamental frequency of the power system, spectral leakages occur and these leakages can be referred to as interharmonics. Such spectral leakage can be reduced using windowing methods, such as Hanning and Hamming [3,4].

According to the standard 61000-4-7 specified by IEC, for the $50 \mathrm{~Hz}$ mains frequency, the signal whose DFT will be taken is proposed to be windowed with 10 cycles $(200 \mathrm{~ms})$. However, this windowing complicates the application and analysis [5]. To reduce the effect of spectral leakage based on the structure of DFT, changing the frequency resolution was proposed in $[6,7]$. However, in this method, optimization is needed for adjusting frequency scale according to the signal.

In the study carried out in [8], the spectral leakage effect around the fundamental frequency was calculated and then it was filtered out from the DFT of the signal. Thus, the spectral leakage effect was eliminated. However, in this study, the fundamental frequency is assumed to be the component with the highest amplitude. Analysis was performed only for the interharmonics close to the fundamental frequency.

In the other study carried out by [9], where interharmonics are close to harmonic frequency, calculation results of both harmonic and interharmonic may be incorrect because of the spectral leakage and also the picket-fence effect occurring at the $5 \mathrm{~Hz}$ resolution suggested by IEC.

MUSIC and ESPRIT provide the high frequency resolution demanded for the analysis of the harmonic and interharmonic components [10]. But, these methods need a prefilter 
to be able to catch the fundamental frequency and success of the methods depends on the accurate calculation of the fundamental frequency.

Kalman filter was used for the harmonic analysis in [11, 12]. The most important disadvantage of the Kalman filter is a high ordered model requirement for the determination of the harmonic components [13]. Presents a method which uses PLL based filter bank that measures harmonic-interharmonic amplitude and phase values for the case of changing fundamental frequency. PLL used in [13] is successful for synchronizing with fundamental frequency. However, because of the use of the band pass filter and fixed central frequency, the existence of the interharmonics whose frequencies are close harmonics may lead to leakages.

In another study [14] in which PLL based adaptive notch filter is used, amplitudes and frequencies of the harmonic and interharmonic were calculated. Because the response rate of the proposed method is high, it is suitable for real time applications. However, performance analysis of the proposed method for the interharmonics was realized by selecting a frequency not close to the fundamental and harmonic components. Digital PLL proposed in [15] was compared with available PLL methods in the literature in terms of the cases including phase shifting, voltage sag, harmonic, frequency change, and multizero crossing. Its superiority was shown with the support of experimental studies.

PLL used in our study has the same structure with that in [15]. In our study, a PLL based digital adaptive filter is proposed to measure the interharmonics. It is also shown in [15] that the software based PLL structure used in this method has superiority over the other PLL structures.

It is shown in the study [16] that notch filter bank used in the proposed PLL based measurement system and notch filter structure are adaptive to the frequency change of the signal whose fundamental component frequency is measured. Furthermore, because band width is adjustable, it can be kept in a narrow band. This prevents the interharmonic leakage with the frequency close to that of the harmonic frequency components [13].

The proposed method is proper for the offline calculations. Meanwhile, it also works for the cases such as phase shifting and multizero crossing together with amplitude and frequency change $[14,18]$. Furthermore, the proposed method was tested for the interharmonics close to the fundamental frequency and harmonic frequencies and it generated more successful results comparing to the calculationmethods 3 suggested in IEC-61000-4-7 [17].

When cases of multizero crossing, phase shifting, frequency changing, and voltage sag, especially, occur synchronously, the proposed method gives better results than FFT and DFT. That is a new study in the literature to determine the interharmonics.

This paper is organized as follows. In Section 2, the proposed method was introduced. In Section 3, the proposed method is tested for the interharmonics close to the fundamental component and harmonic component. Furthermore, experimental studies were performed for the cases of frequency change, amplitude change, and phase shifting that often occur in energy systems and also the case of the multizero crossing about which there has been few studies in the literature. In Section 4, the method is compared with the studies in the literature and the available standards.

\section{Proposed Method}

The structure of the proposed method has three blocks as shown in Figure 1. The block A represents the software based PLL structure. A power signal including various disturbances like harmonic, frequency changing, amplitude changing, phase shifting, and multizero crossing is applied to PLL as input. PLL locked up the fundamental component $\left(w_{0}\right)$ of the power signal in input and generates the signals specified as integer power of $w_{0}$ like $\sin w_{0}, \sin 2 w_{0}, \sin 3 w_{0}, \ldots, \sin n w_{0}$, $\cos w_{0}, \cos 2 w_{0}, \cos 3 w_{0}, \ldots, \cos n w_{0}$ in output. PLL is very sensitive to changes in fundamental frequency.

The block B consists of number " $n$ " of adaptive notch filters whose suppression frequency is adjustable and suppresses the fundamental components frequency of the signal applied to each adaptive notch filter. The signal frequencies, $\sin n w_{0} / \cos n w_{0}$, which are applied to the notch filter, are the notch filter suppression frequency as well. In summary, if the fundamental frequency of the signal applied to the notch filter input/enter changes, the notch filter suppression frequency also changes. In each filter output, the components of the input signal, $A n \cdot \sin n w_{0} / B n \cdot \cos n w_{0}$ are suppressed. Subtracting each filter output from the input signal gives the instant change in all interharmonics which occurred in the input signal in the output of the block B. Regarding IEC standards, FFT of the signal with interharmonics, which is obtained from the adaptive filter output in the block $\mathrm{C}$, are taken. Thus, amplitudes and frequencies of the interharmonics in the signal can be calculated separately and correctly.

2.1. Software Based PLL. PLL is a closed-loop servosystem, which continuously controls the output signal to minimize the difference between the input and the output signals; PLL aims to lock the frequency and phase of the feedback signal, $x_{(k)}$, to the phase and frequency of the input signal, $V_{(t)}$.

Structure of the software based PLL that is used in the proposed method for the interharmonic analysis is shown in Figure 2. The used PLL structure is the same with the structure of the developed PLL in [15]. The PLL structure, as shown in Figure 2, consists of 4 blocks, namely, phase detector (PD), frequency divider (FD), PI, and voltage controlled oscillator (VCO). The superiority of the proposed PLL over some structures (like pPLL, parkPLL ve EPLL) widely used in the literature and its success in the cases including some disturbances like phase shifting, amplitude changing, and multizero crossing were shown with the support of the results obtained from the real time experiments [15]. Estimation of the fundamental frequency in the harmonic and interharmonics analysis is very important. Even a small error made in the estimation process can bring about considerable calculation mistakes. Therefore, PLL must quickly lock in the fundamental component of the signal in the cases including one or more disturbances and it must be robust against disturbances. They play an important role in the accuracy of the analysis. 


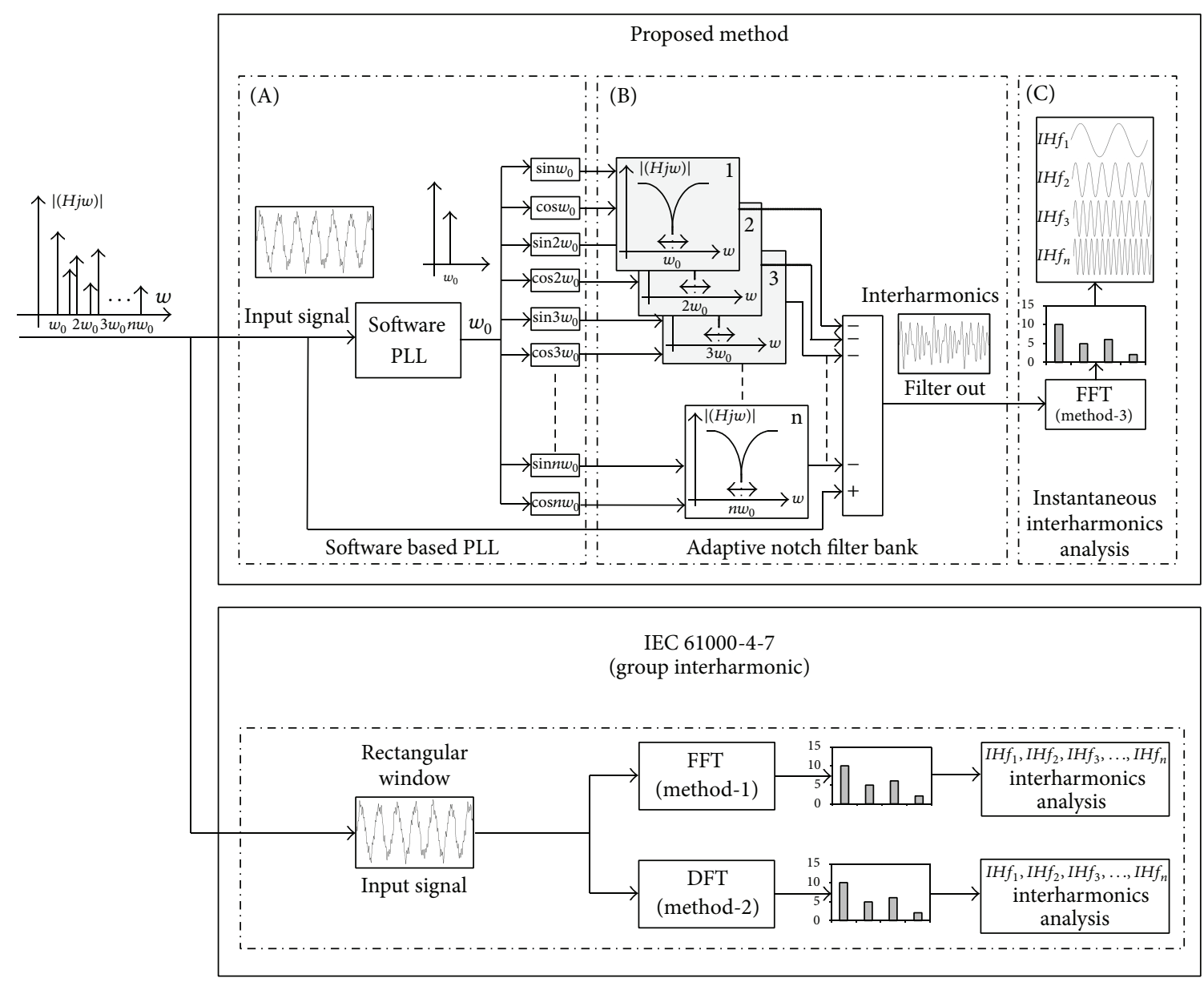

FIGURE 1: Block structure of the proposed method and IEC 61000-4-7 group method.

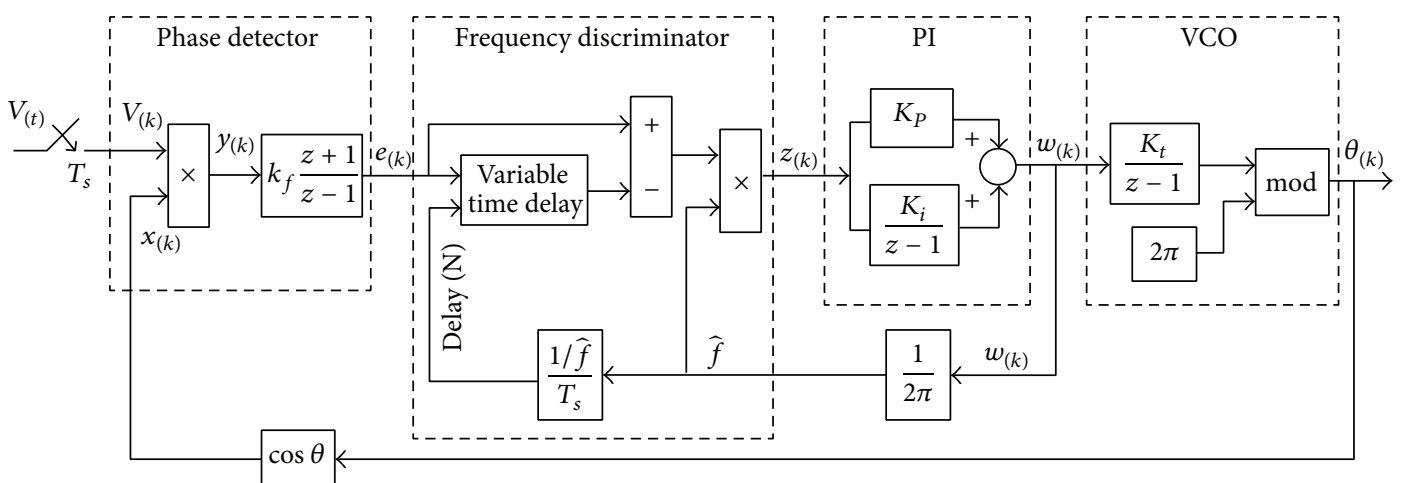

FIGURE 2: The phase locked loop (PLL) block diagram.

It is shown with the experimental studies that the PLL structure shown in Figure 2 is robust against each one of the disturbances such as phase shifting, amplitude changing, frequency changing, and multizero crossing [15]. In all studies in the literature as well as in [15], the response performance of PLL was examined considering the cases including only one disturbance. In this study, in the analysis of the digital PLL based interharmonic, the studies were made considering the cases including 4 disturbances at the same time and then accuracy of the proposed measurement structure and its stability were shown.
2.2. Digital Adaptive Notch Filter. Calculation of the discretetime transfer function for the notch filter proposed in the study and frequency response analysis are given in [16]. Transfer function of the notch filter (shown as bold in Figure 3), which damps the angular frequency, is given in the following:

$$
H\left(e^{j w T}\right)=\frac{\cos w_{\Delta} T+j \sin w_{\Delta} T-1}{\left\{1+2 K V^{2}\right\}\left\{\cos w_{\Delta} T+j \sin w_{\Delta} T\right\}+2 K V^{2}-1},
$$




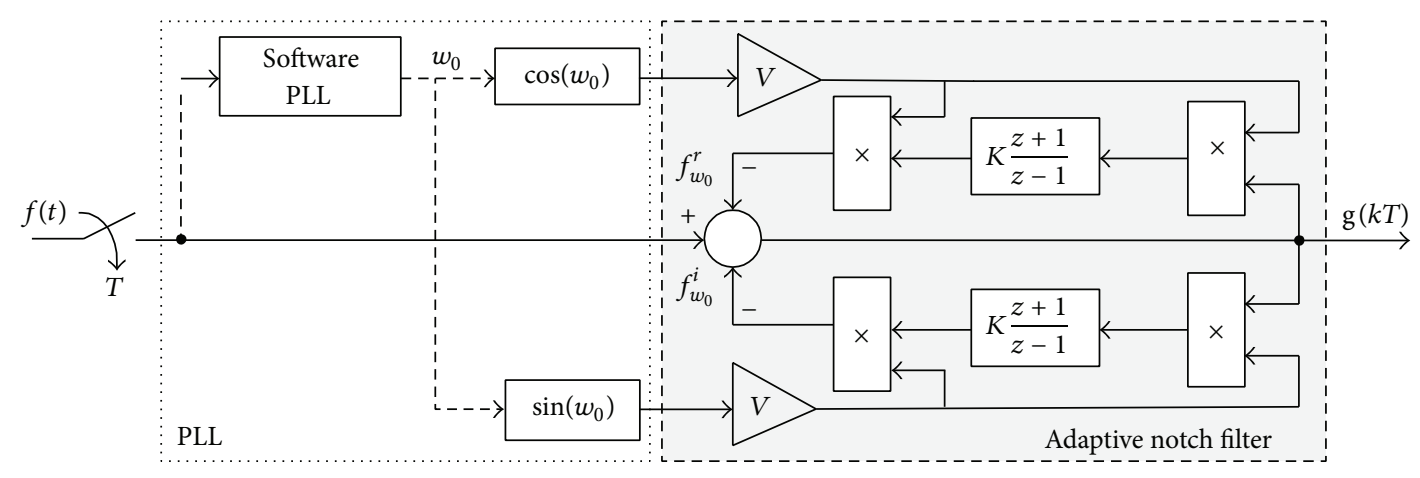

FIGURE 3: Digital adaptive notch filter block diagram.
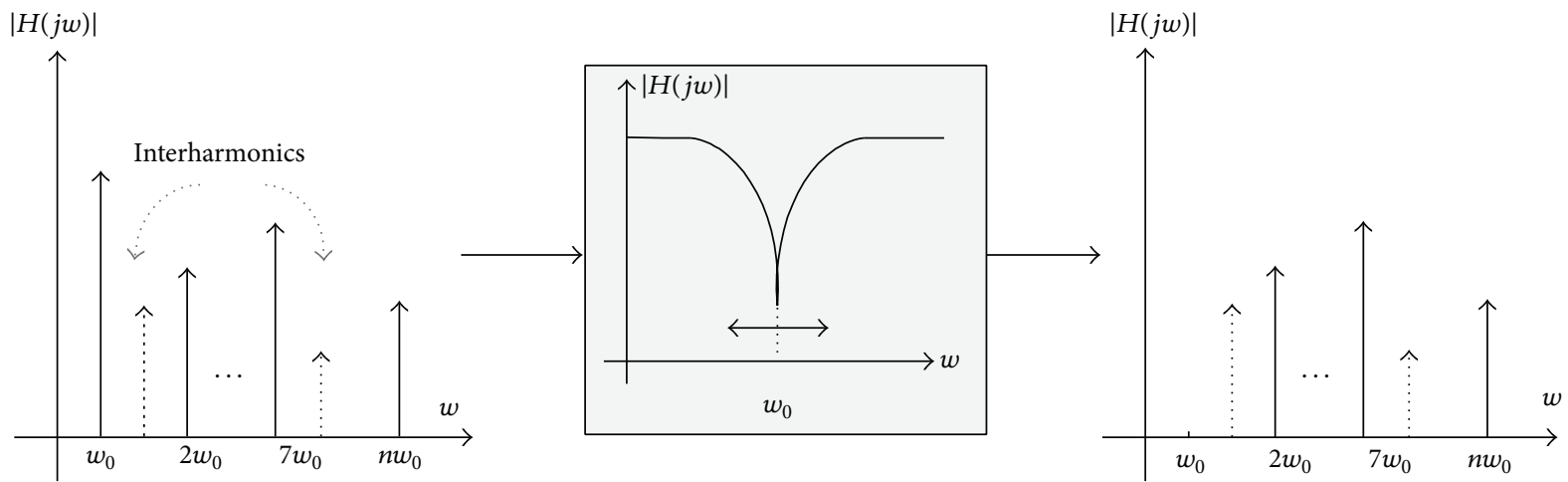

FIGURE 4: The response of the notch filter.

where $T$ represents the sampling time, $w_{0}$ is the angular frequency of the reference signal for $w_{\Delta}=w-w_{0}, w$ represents the angular frequency of the signal $f(t)$, the amplitude of the reference signals, $\sin w_{0}$ and $\cos w_{0}$, is represented as $V$, and lastly $K$ represents the integrator factor. The frequency response of $H\left(e^{j w T}\right)$ belonging to the adaptive notch filter is shown in Figure 4. The signal applied to the input of the adaptive notch filter is comprised of the fundamental component $\left(w_{0}\right)$, harmonic components $\left(2 w_{0}, 3 w_{0}, 4 w_{0}, \ldots, n w_{0}\right)$, and interharmonic components. In addition, it is seen that $w_{0}$ is suppressed in the output of the filter.

The angular frequency, $w_{0}$, of the reference signals, $\sin w_{0}$ and $\cos w_{0}$, determines the suppression frequency of the notch filter. If the angular frequency, $w_{0}$, of the reference signals changes, the suppression frequency of the notch filter equals the changing frequency. Since a notch filter whose suppression frequency can be adjusted with respect to the input reference signal angular frequency is obtained, such a filter is called adaptive notch filter.

\section{Simulation Results}

In this section, the performance of the proposed method is tested considering the simulations made using MATLAB and the real data taken from the arc furnace. Accuracy of the proposed method and its superiority are shown with the simulations made considering different mains conditions and disturbances (such as frequency changing, phase changing,
TABLE 1: PLL and adaptive notch filter coefficients.

\begin{tabular}{lccc}
\hline \multicolumn{1}{c}{ PLL } & \multicolumn{2}{c}{ Adaptive filter } \\
Coefficient & Value & Coefficient & Value \\
\hline$K_{i}$ & 1800 & $K$ & 0.0085 \\
$K_{p}$ & 60 & $V$ & 0.125 \\
$K_{t}$ & 1.0 & $T_{s}$ & $50 \mu \mathrm{s}$ \\
$T_{s}$ & $50 \mu \mathrm{s}$ & & \\
\hline
\end{tabular}

multizero crossing, and voltage sag) for $T_{s}=50 \mu \mathrm{s}$. Amplitude of the fundamental component in the generated signal is considered $1 \mathrm{pu}$ and amplitudes of the interharmonics are selected as 0.05 and $0.1 \mathrm{pu}$. Selecting the interharmonic amplitudes lower than the fundamental component amplitude in the determination of the interharmonics is realized due to satisfying the filter accuracy and sensitivity. Table 1 includes the coefficients about the software based PLL used in the proposed method and the adaptive notch filter.

The standard FFT (Method 1) and DFT (Method 2) suggested by IEC are used to analyze these signals including both harmonic and also interharmonic components. Furthermore, according to IEC standards, FFT (Method 3) is used to analyze the signal with interharmonics, which is obtained from the adaptive filter output. $N=1000$ samples are taken for the FFT analysis and in the DFT analysis, IEC 61000-4-7 standards (which suggest using the windows with the period 


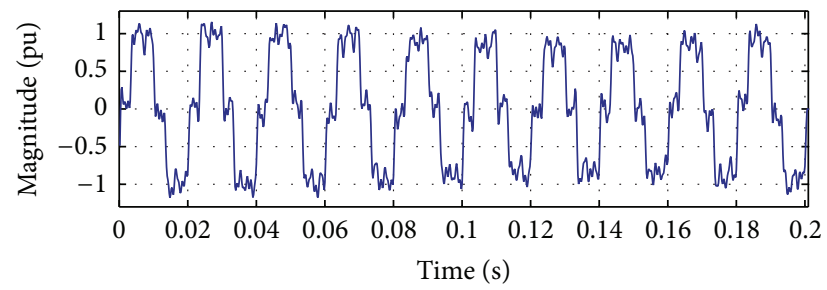

FIgURE 5: The signal $x(t)$ for $f=50 \mathrm{~Hz}$ and $T=0.2 \mathrm{~s}$.

of $T=10 / f$ at the $5 \mathrm{~Hz}$ resolution for $f=50 \mathrm{~Hz}$ ) are considered. Signals are windowed with a rectangular window and interharmonic group method is used to calculate the interharmonic with FFT and DFT.

3.1. Case I: Interharmonic Close to the Fundamental Frequency. In the first case, the signal $x(t)$ which will be analyzed consists of 4 interharmonics and 6 harmonics. The component, $1.06 \omega t$, from interharmonic is selected too close to the fundamental frequency component. In most of the studies in the literature, it is obviously seen that interharmonic frequencies were generally selected far away from the fundamental and harmonic frequency. This provides a calculation convenience for the methods based on FFT and DFT, too.

However, like in the signal specified with (2), interharmonics in a real power signal may be close to fundamental component. The input signal, $x(t)$, is shown in Figure 5. There is multizero crossing as a disturbance in the signal. Consider

$$
\begin{aligned}
x(t)= & \sin (\omega t)+0.2 \cdot \sin (5 \omega t)+0.14 \cdot \sin (7 \omega t) \\
& +0.08 \cdot \sin (11 \omega t)+0.06 \cdot \sin (13 \omega t) \\
& +0.03 \cdot \sin (17 \omega t)+0.08 \cdot \sin (19 \omega t) \\
& +0.1 \cdot \sin (1.06 \omega t)+0.05 \cdot \sin (3.4 \omega t) \\
& +0.1 \cdot \sin (10.6 \omega t)+0.05 \cdot \sin (11.7 \omega t) .
\end{aligned}
$$

In the signal $x(t)$ in (2), for $f=50 \mathrm{~Hz}$, harmonic frequencies are 250,350,550,650,850, and $950 \mathrm{~Hz}$ and interharmonic frequencies are 53, 170, 530, and $585 \mathrm{~Hz}$. In Figure 6, (a) DFT analysis of the signal $x(t)$ at $5 \mathrm{~Hz}$, (b) FFT analysis of the signal $x(t)$, (c) frequency spectrums of FFT analysis of the proposed filter output signal, and (d) reference-filter output signals are shown.

$F_{s}$ is the sampling frequency and $N$ is the number of samples, If the frequency of the analysis harmonic component is not integer power of $F_{s} / N$, the signal power which must center on the only one frequency point goes to the other neighboring frequency points and a loss occurs. This is expressed as "picket-fence effect" or "notch loss" in the literature [18].

In Figures 6(b) and 6(c), this situation mentioned above realizes in the DFT and FFT analysis. In the analysis, the rate $F_{s} / N$ is $5 \mathrm{~Hz}$ because $F_{s}=5000$ and $N=1000$. Since the interharmonic component selected close to the fundamental frequency, with $53 \mathrm{~Hz}$, is not integer power of FFT and DFT points with $5 \mathrm{~Hz}$, a flow from this frequency component to
$50 \mathrm{~Hz}$ and $55 \mathrm{~Hz}$ occurs. In such a case, the interharmonic analysis methods based on DFT and FFT may result in large calculation errors. An unreal interharmonic can come out due to this spectral dispersion, and vice versa.

In Figures 6(b) and 6(c), due to the spectral dispersion, interharmonic component of $53 \mathrm{~Hz}$ can be considered as a sidelobe of the fundamental component with $50 \mathrm{~Hz}$. This situation may create a problem for adjacent two harmonic components. In the proposed method, adaptive notch filter suppresses the fundamental component $w_{0}$ and all harmonic components, $n w_{0}$ for $n=1,2,3, \ldots$, after an accurate synchronization between software based PLL and the fundamental frequency in the signal are provided. Thus, only instant total of interharmonics is obtained in the filter output. In Figure 6(a), examining the FFT spectrum of the filter output, it is underlined that small sidelobes in the interharmonic with $53 \mathrm{~Hz}$ occur. However, since there is not a neighboring frequency component, FFT results of the filter output signal calculate the amplitude value with high accuracy.

An analysis is carried out to determine interharmonics in the signal $x(t)$ for three fundamental frequency values, 49.5, 50 , and $50.5 \mathrm{~Hz}$. The results are given in Table 2. Values shown with bold characters in Table 2 give the analysis results for the three fundamental frequencies, $49.5,50$, and $50.5 \mathrm{~Hz}$ which belong to the interharmonic component with $1.06 \omega t$. When examining results of the analysis realized for the fundamental frequency, $f=50 \mathrm{~Hz}$. Error values of the proposed method, DFT, and FFT are $0.94 \%, 3.81 \%$, and $3.03 \%$, respectively. Errors in method 1 and method 2 are higher than that in the proposed method due to the leakages depending on the "picket-fence" and "windowing." DFT results have high calculation mistakes for the fundamental frequency values like $f=$ $49.5 \mathrm{~Hz}$ and $50.5 \mathrm{~Hz}$. However, for other two selected interharmonics, it gives better results than FFT and DFT as well. Analysis results of Case 1 validate that the proposed method gives better results than FFT and DFT.

3.2. Case 2: Interharmonic Analysis under Multiple Disturbances. In power systems, voltage sag, frequency changing, phase shifting, multizero crossing, and other disturbances occur due to various reasons. In harmonic and interharmonic analysis, exactly what time disturbance occurs is not mostly known. However, even if most of the disturbances occur simultaneously, it is important to determine the fundamental frequency value accurately or to synchronize with the fundamental frequency well. In the literature, there have been studies in which different methods are used for disturbances. 


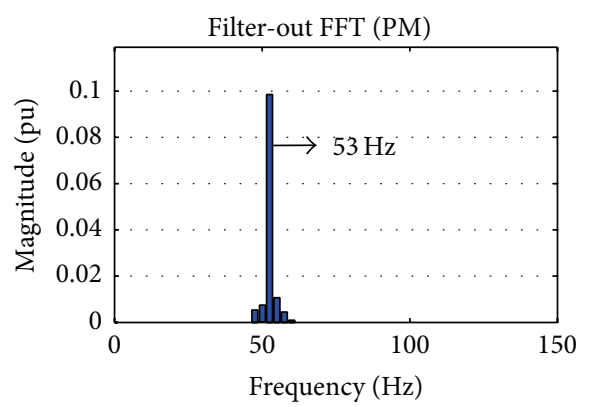

(a)

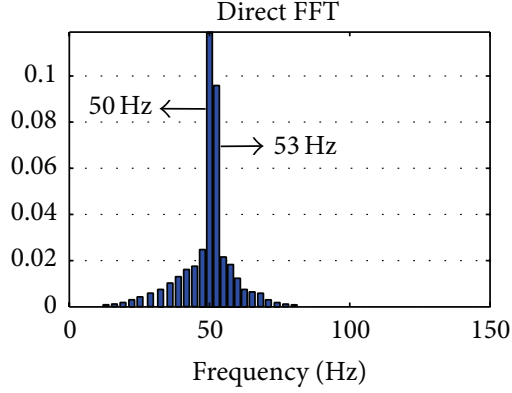

(b)

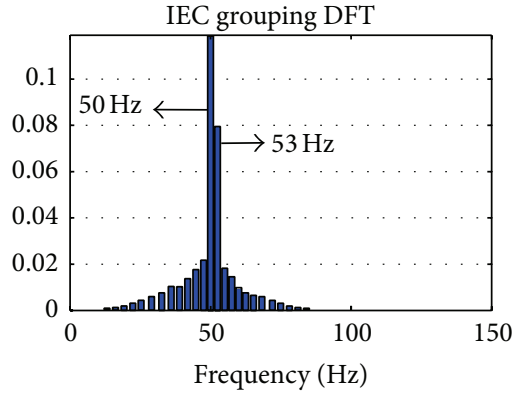

(c)

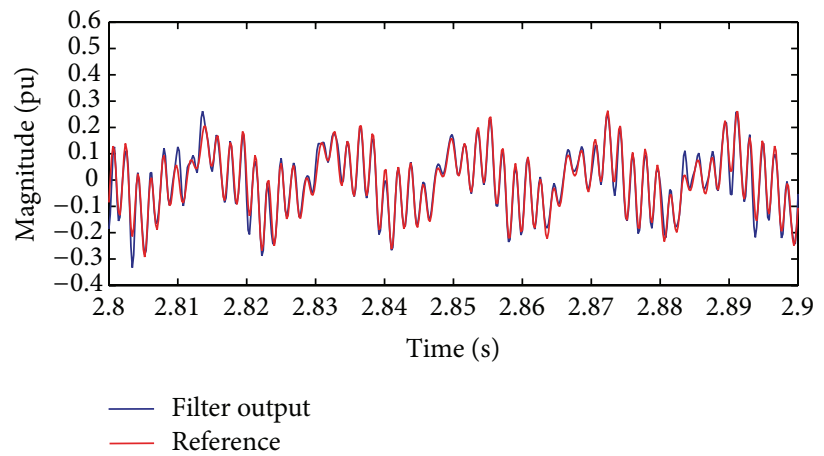

(d)

Figure 6: $f=50 \mathrm{~Hz}, T=0.2 \mathrm{~s}$, and $N=1000$ samples; (a) FFT for the filter output, (b) FFT for the signal $x(t)$, (c) DFT frequency spectrums for the signal $x(t)$, and $(\mathrm{d})$ reference-filter output signals.

TABLE 2: Simulation results for Case 1.

\begin{tabular}{ccccccccc}
\hline$f(\mathrm{~Hz})$ & Interharmonics & Actual value & FFT (method-1) & Error (\%) & IEC (method-2) & Error (\%) & PM (method-3) & Error (\%) \\
\hline \multirow{4}{*}{49.5} & $1.06 \times f$ & 0.1 & 0.0963 & $\mathbf{3 . 6 7 9}$ & 0.1124 & $\mathbf{1 2 . 4 1}$ & 0.0974 \\
& $3.40 \times f$ & 0.05 & 0.0499 & $\mathbf{0 . 2 8 1}$ & 0.0477 & $\mathbf{4 . 5 7 2}$ & 0.0500 & $\mathbf{0 . 0 4 2}$ \\
& $10.60 \times f$ & 0.1 & 0.0995 & $\mathbf{0 . 5 4 2}$ & 0.0102 & $\mathbf{2 . 8 8 6}$ & 0.0999 & $\mathbf{0 . 0 2 8}$ \\
& $11.70 \times f$ & 0.05 & 0.0497 & $\mathbf{0 . 6 0 1}$ & 0.0505 & $\mathbf{1 . 0 0 5}$ & 0.0499 & $\mathbf{0 . 0 3 7}$ \\
\hline \multirow{4}{*}{50} & $1.06 \times f$ & 0.1 & 0.0970 & $\mathbf{3 . 0 3 7}$ & 0.0961 & $\mathbf{3 . 8 1 0}$ & 0.0990 & $\mathbf{0 . 9 4 8}$ \\
& $3.40 \times f$ & 0.05 & 0.0500 & $\mathbf{0 . 0 0 0}$ & 0.0500 & $\mathbf{0 . 0 0 0}$ & 0.0500 & $\mathbf{0 . 0 2 6}$ \\
& $10.60 \times f$ & 0.1 & 0.1000 & $\mathbf{0 . 0 0 0}$ & 0.1000 & $\mathbf{0 . 0 0 0}$ & 0.0999 & $\mathbf{0 . 0 1 8}$ \\
& $11.70 \times f$ & 0.05 & 0.0500 & $\mathbf{0 . 0 0 0}$ & 0.0500 & $\mathbf{0 . 0 0 0}$ & 0.0499 \\
\hline \multirow{4}{*}{50.5} & $1.06 \times f$ & 0.1 & 0.0962 & $\mathbf{3 . 8 3 3}$ & 0.0837 & $\mathbf{1 6 . 2 3}$ & 0.0974 \\
& $3.40 \times f$ & 0.05 & 0.0499 & $\mathbf{0 . 1 7 2}$ & 0.0505 & $\mathbf{0 . 9 9 6}$ & 0.0500 & $\mathbf{0 . 0 4 5}$ \\
& $10.60 \times f$ & 0.1 & 0.0992 & $\mathbf{0 . 7 8 0}$ & 0.1029 & $\mathbf{2 . 8 6 6}$ & 0.1000 & $\mathbf{0 . 0 2 7}$ \\
& $11.70 \times f$ & 0.05 & 0.0495 & $\mathbf{0 . 9 3 7}$ & 0.0481 & $\mathbf{3 . 7 5 7}$ & 0.0500 & $\mathbf{0 . 0 3 9}$ \\
\hline
\end{tabular}

However, it is assumed that one or more disturbances occurred in different times in the study. As we know, most of these disturbances can occur in a power system simultaneously. For this reason, considering that such disturbances can occur simultaneously, they are formed simultaneously in this section. Performance of the proposed method and other methods is tested by using the signal expressed in in the following:

$$
\begin{aligned}
x(t)= & \sin (\omega t)+0.2 \cdot \sin (5 \omega t)+0.14 \cdot \sin (7 \omega t) \\
& +0.08 \cdot \sin (11 \omega t)+0.06 \cdot \sin (13 \omega t)
\end{aligned}
$$

$$
\begin{aligned}
& +0.03 \cdot \sin (17 \omega t)+0.08 \cdot \sin (19 \omega t) \\
& +0.1 \cdot \sin (5.5 \omega t)+0.05 \cdot \sin (7.4 \omega t) \\
& +0.1 \cdot \sin (11.6 \omega t)+0.05 \cdot \sin (13.8 \omega t)
\end{aligned}
$$

In the signal $x(t)$ expressed in (3), for $f=50 \mathrm{~Hz}$, full harmonic frequencies are 250, 350, 550, 650, 850, and $950 \mathrm{~Hz}$ and interharmonic frequencies are 275, 370, 580, and $690 \mathrm{~Hz}$. In Figure 10, values and formation times of 4 disturbances are shown. In Figure 7(a), for $T=3 \mathrm{~s}$, the fundamental frequency value is increased from $50 \mathrm{~Hz}$ to $52.5 \mathrm{~Hz}$ with 


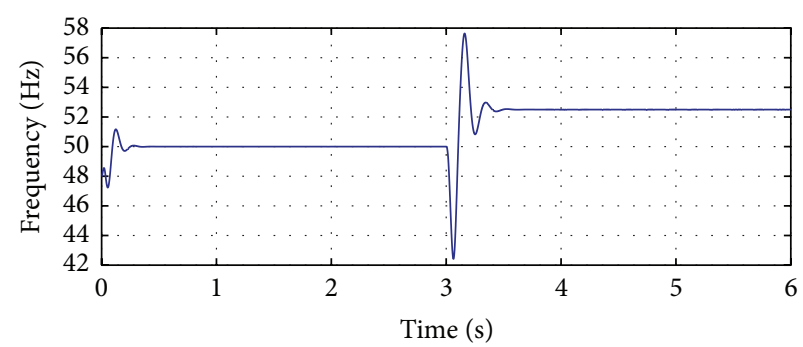

(a)

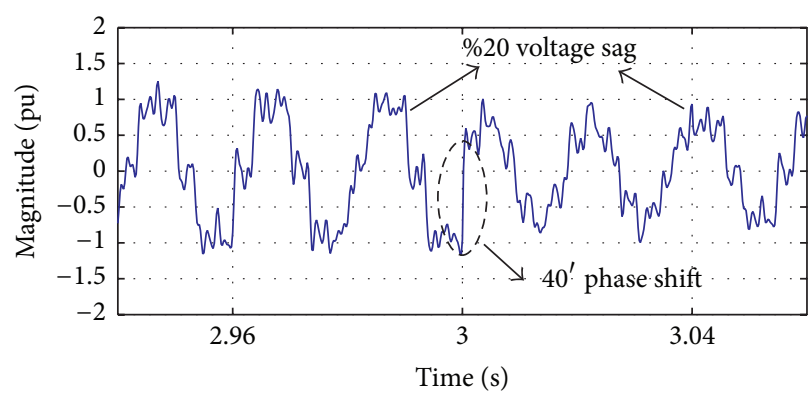

(b)

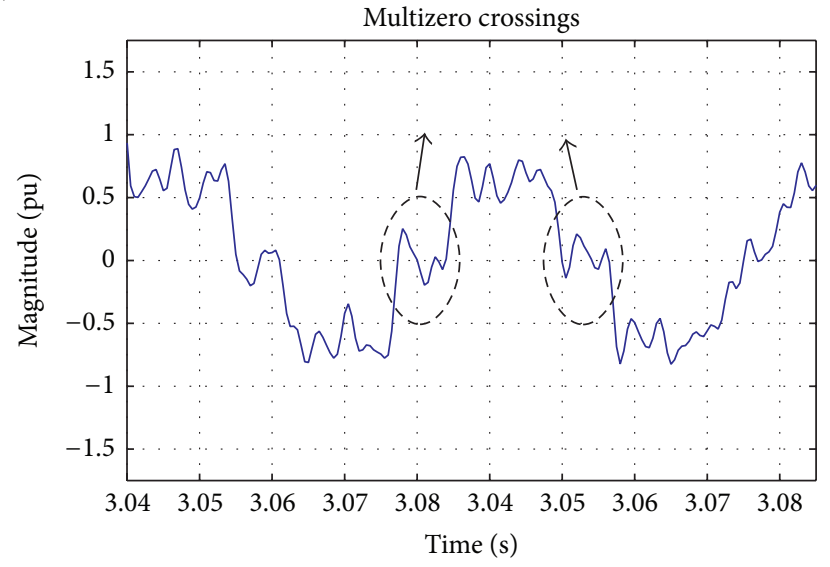

(c)

Figure 7: The signal, $x(t)$, under multiple disturbances; (a) $+2.5 \mathrm{~Hz}$-frequency change, (b) $20 \%$-voltage sag and $40^{\circ}$-phase shifting, and (c) multizero crossing.

the change of $+2.5 \mathrm{~Hz}$. In a power system in which the fundamental frequency is $50 \mathrm{~Hz}$, the $2.5 \mathrm{~Hz}$ frequency change $\left(\Delta f_{o}=5 \%\right)$ is not a most commonly encountered situation.

Although such a frequency change is $\pm 1 \%( \pm 0.5 \mathrm{~Hz})$, a great value like $+5 \%$ is selected. In Figure $7(\mathrm{~b})$, formation time of the $40^{\circ}$ phase shifting and $20 \%$ voltage sag is $T=3 \mathrm{~s}$, too. In Figure $7(\mathrm{c})$, multizero crossing which has the different disturbances is seen. In Figure 8(a), the $x(t)$ signal under multi disturbant effect is seen, in Figure 8 (b) the reference and filter out signals are seen and in Figure 8(c) the FFT spectrum of the filter out signal is seen. Voltage sag usually occurs in the first operation/activation/initiation of large powered machines and systems, which lasts less than 1 minute and affects other running systems and devices negatively.

Multizero crossing is usually formed by high ordered harmonic or interharmonic components. This causes problems while both calculating the fundamental frequency and also synchronizing with the fundamental frequency. Furthermore, algorithms [7] depending on the assumption in which the signal passes from only one zero point are used to determine accurate multizero crossing and zero crossing point. But they cause calculation mistakes.

Analysis results are given in Table 3 focusing on the results in Table 3; maximum error of the proposed filter which is tested under the simultaneously occurring disturbances is $0.379 \%$.

Comparing to the other methods, maximum errors of FFT and DFT are $2.52 \%$ and $4.52 \%$, respectively. Error values in FFT and DFT analysis are high due to the spectral leakage and picket-fence effect the disturbance leads to.

With Case 3, it is shown that the proposed method has superiority over the other methods considering the simultaneously occurring disturbances like frequency changing, voltage sag, phase shifting, and multizero crossing. For 3 different cases that were formed considering different mains situations and disturbances, using the proposed method in this study and other methods, analyses are made and the following results can be obtained.

In the analysis of a signal including an interharmonic close to the fundamental frequency or full harmonic frequency, "picket fence" and "spectral leakage" can occur in the noninteger values of the fundamental frequency. Due to these effects, calculation methods suggested by IEC cannot provide sufficient resolution in the interharmonic analysis. To reduce these effects, increasing the resolution and using one of the windowing methods are needed. For interharmonic analysis which is realized under the multiple disturbances like multizero crossing firstly being applied in this study in the literature, phase shifting, frequency changing, and voltage sag, it is seen that FFT and DFT methods make high calculation errors.

"Spectral leakage" and "picket-fence" cause both inaccurate calculation of interharmonics and also sometimes occurrence of an unreal harmonic. In the proposed method, since the signal obtained from the filter output contains only interharmonic frequency components, interharmonics 

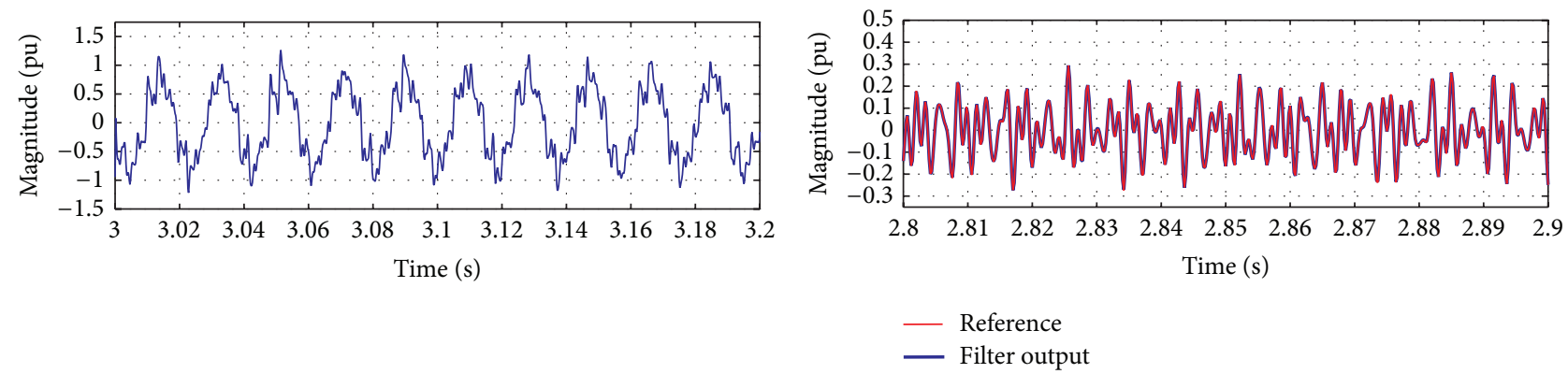

(a)

(b)

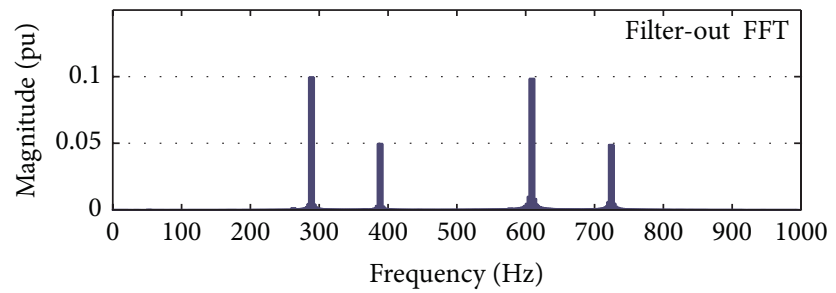

(c)

FIGURE 8: Under the multiple disturbances; (a) the signal, $x(t)$, (b) reference-filter output signal, and (c) FFT of the filter output.

TABLE 3: Simulation results for Case 2.

\begin{tabular}{ccccccccc}
\hline$f(\mathrm{~Hz})$ & Interharmonics & Actual value & FFT (method-1) & Error (\%) & IEC (method-2) & Error (\%) & PM (method-3) & Error (\%) \\
\hline & $5.50 \times f$ & 0.1 & 0.0979 & $\mathbf{2 . 1 3 8}$ & 0.0955 & $\mathbf{4 . 5 2 3}$ & 0.0999 & $\mathbf{0 . 0 9 7}$ \\
52.5 & $7.40 \times f$ & 0.05 & 0.0495 & $\mathbf{0 . 9 7 0}$ & 0.0512 & $\mathbf{2 . 3 0 2}$ & 0.0500 & $\mathbf{0 . 0 6 8}$ \\
& $11.60 \times f$ & 0.1 & 0.0992 & $\mathbf{0 . 7 5 6}$ & 0.1043 & $\mathbf{4 . 2 6 1}$ & 0.0998 \\
& $13.80 \times f$ & 0.05 & 0.0487 & $\mathbf{2 . 5 2 1}$ & 0.0512 & $\mathbf{2 . 3 3 6}$ & 0.0498 & $\mathbf{0 . 3 7 9}$ \\
\hline
\end{tabular}

can be calculated more accurately comparing to the other methods.

3.3. Case 3: Test of the Proposed Method through Real Data. To test the performance of the proposed method using the real data, the data was first taken from an arc furnace driven by a $350 \mathrm{kVA}$ frequency converter. The arc furnace realizes the melting process at a frequency of $50 \mathrm{~Hz}$. It was taken at a sampling frequency of $5 \mathrm{kHz}$ for 13 seconds while the arc furnace was running in full capacity.

In Figure 9, (a) current wave form of the arc furnace and (b) FFT spectrum of the current signal for $N=2000$ samples are indicated. The signal is firstly windowed with rectangular window and then its corrected FFT is taken. In the frequency spectrum of FFT, interharmonics having the amplitudes of 8.145 $\mathrm{A}$ and $7.938 \mathrm{~A}$ are determined at the frequencies of 1658 and $1758 \mathrm{~Hz}$. These amplitude values are found as 3.04\% and $2.96 \%$ of the fundamental component.

Furthermore, fundamental component current is $267.5 \mathrm{~A}$ and current amplitudes for six harmonics 5th, 7th, 11th, 13th, 17 th and 19th harmonic are calculated as 52.05, 29.29, 15.87, $10.88,6.78$, and $5.04 \mathrm{~A}$, respectively. In Figure 10, (a) the proposed filter output signal for the current of the arc furnace and (b) FFT spectrum of the current signal for $N=2000$ samples are shown.

In the frequency spectrum of FFT, interharmonics having the amplitudes of $8.127 \mathrm{~A}$ and $7.925 \mathrm{~A}$ are determined at the frequencies of $1658 \mathrm{~Hz}$ and $1758 \mathrm{~Hz}$. Apart from these, for 4 frequencies, namely, 1457.5, 1535, 1635, and $1957.5 \mathrm{~Hz}$, interharmonics with low amplitude are generated and they are $1.319,1.355,1.444$, and $1.229 \mathrm{~A}$, respectively.

In the proposed filter output, interharmonics with different frequency values less than $1 \mathrm{~A}$ are determined, but they are ignored in the calculation because their amplitudes are so small. Analysis results in Table 4, it seems that the proposed method is suitable for the real data analysis.

\section{Conclusion}

In this study, PLL based digital adaptive filter was proposed for measuring the interharmonics. In the PLL based measuring system, filter banks were formed using a lot of notch filters. Furthermore, due to the superiority of the software based PLL, it provides accurate synchronization with the fundamental frequency.

Filter is adaptive against the change in the fundamental frequency of the signal and filter bandwidth is adjustable. Keeping the band width in a short range prevents the leakages resulting especially from interharmonics close to the full harmonics. With the proposed method, the signal is including amplitude, phase angles, active-reactive current components, and instant total interharmonics belonging to each harmonic. And then, without leakage effects of harmonics, by means of FFT analysis of the signal, frequency, phase, and amplitudes 


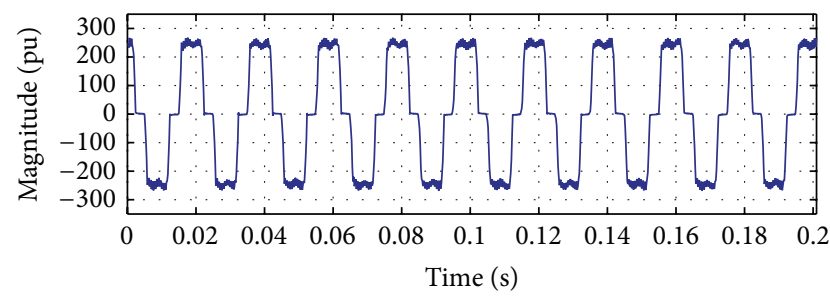

(a)

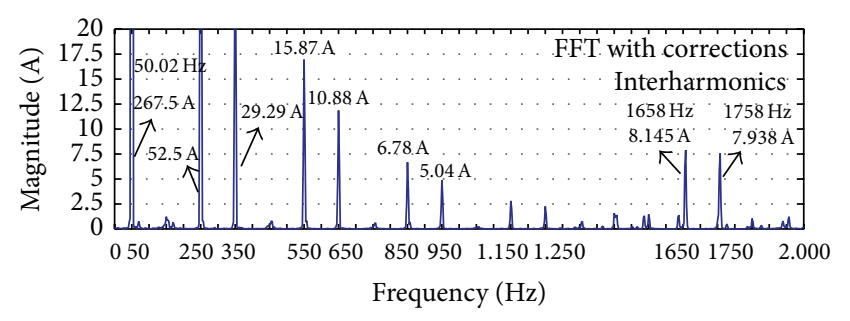

(b)

Figure 9: (a) The arc furnace current signal for $T=0.2 \mathrm{~s}$; (b) FFT of the arc furnace current signal.

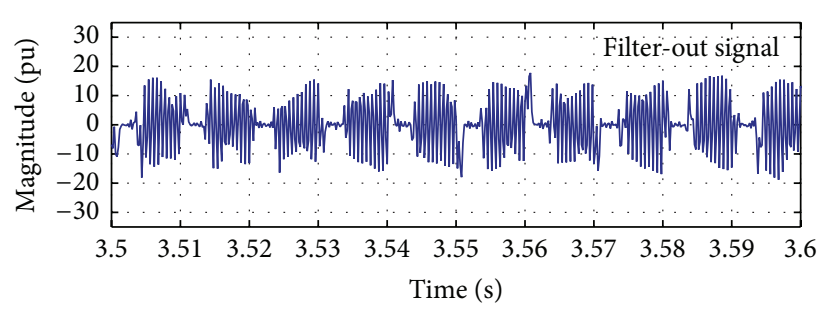

(a)

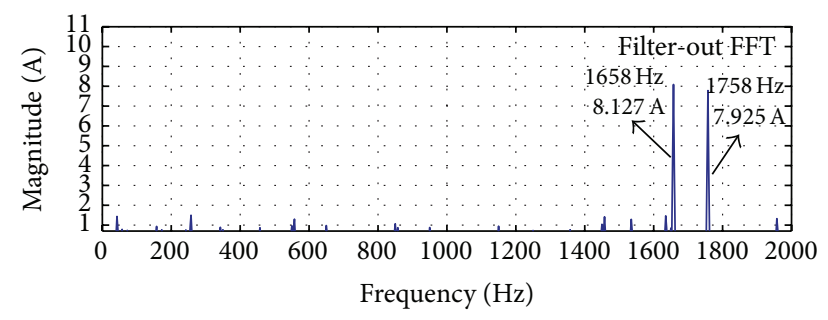

(b)

Figure 10: (a) Filter output signal for $T=0.1 \mathrm{~s}$; (b) FFT of the filter output signal.

TABLE 4: Analysis results obtained with the area data.

\begin{tabular}{cccccc}
\hline$f(\mathrm{~Hz})$ & Interharmonic frequency $(\mathrm{Hz})$ & FFT with correction (reference) & Error (\%) (reference) & Proposed method & Error (\%) \\
\hline & 1457.5 & 1.309 & 0.000 & 1.319 & 0.7639 \\
50.02 & 1535 & 1.348 & 0.000 & 0.5192 \\
& 1635 & 1.445 & 0.000 & 0.0692 \\
& 1658 & 8.145 & 0.000 & 1.444 & 8.127 \\
& 1758 & 7.938 & 0.000 & 7.925 & 0.1637 \\
& 1957.5 & 1.238 & 0.000 & 0.7269 \\
\hline
\end{tabular}

of each interharmonic are determined more accurately than standards and current studies in the literature.

Furthermore, the proposed method is also tested for interharmonics close to fundamental frequency and primary harmonic frequencies and the obtained results prove that this method is more successful than interharmonic calculation methods in IEC-61000-4-7. Since this method creates no problems like spectral leakage and picket-fence effect, which lead to calculation errors in FFT and DFT, it is more successful comparing to the other methods.

As a new study in the literature to determine the interharmonics, for especially multizero crossing case and also for the cases in which some disturbances like phase shifting, frequency changing, and voltage sag all occurred simultaneously, it gives better results when compared with the other methods.

Analysis results obtained considering the real data taken from the $350 \mathrm{kVA}$ arc furnace are so close to the corrected FFT analysis results used as a reference. As a result, it can be pointed out that this method can be used in the real data analysis. The proposed method is only suitable for offline applications. However, in the future works, it can be suited to the real time applications by increasing the filter response rate.

\section{Conflict of Interests}

The authors declare that there is no conflict of interests regarding the publication of this paper.

\section{References}

[1] C. Li, W. Xu, and T. Tayjasanant, "Interharmonics: basic concepts and techniques for their detection and measurement," Electric Power Systems Research, vol. 66, no. 1, pp. 39-48, 2003.

[2] "Harmonic-interharmonics," Power Quality Application Guide, http://www.copperinfo.co.uk/power-quality/downloads/pqug/ 311-interharmonics.pdf.

[3] A. Testa, D. Gallo, and R. Langella, "On the processing of harmonics and interharmonics: using hanning window in standard framework," IEEE Transactions on Power Delivery, vol. 19, no. 1, pp. 28-34, 2004.

[4] G. W. Chang, C. I. Chen, Y. J. Liu, and M. C. Wu, "Measuring power system harmonics and interharmonics by an improved fast Fourier transform-based algorithm," IET Generation, Transmission and Distribution, vol. 2, no. 2, pp. 192-201, 2008.

[5] M. Yong and W. Bolin, "Research of interharmonic measurement algorithm on power parameters," in Proceedings of the 1st International Conference on Sustainable Power Generation and Supply (SUPERGEN '09), pp. 1-4, April 2009. 
[6] R. C. Wu and T. P. Tsao, "Theorem and application of adjustable spectrum," IEEE Transactions on Power Delivery, vol. 18, no. 2, pp. 372-376, 2003.

[7] T.-P. Tsao, R.-C. Wu, and C.-C. Ning, "The optimization of spectral analysis for signal harmonics," IEEE Transactions on Power Delivery, vol. 16, no. 2, pp. 149-153, 2001.

[8] Ö. Salor, "Spektral leakage elimination of the fourier transform of signals with fundamental frequency deviation," in Proceedings of the 17th Signal Processing and Communications Applications Conference (SIU '09), pp. 852-855, April 2009.

[9] J. Hui, H. Yang, W. Xu, and Y. Liu, "An improved interharmonic grouping scheme based on IEC 61000-4-7," in Proceedings of the 15th International Conference on Harmonics and Quality of Power (ICHQP '12), pp. 178-181, June 2012.

[10] S.-W. Sohn, Y.-B. Lim, J.-J. Yun, H. Choi, and H.-D. Bae, "A filter bank and a self-tuning adaptive filter for the harmonic and interharmonic estimation in power signals," IEEE Transactions on Instrumentation and Measurement, vol. 61, no. 1, pp. 64-73, 2012.

[11] J. A. Macias and A. Gomez, "Self-tuning of Kalman filters for harmonic computation," IEEE Transactins on Power Delivery, vol. 21, no. 1, pp. 501-503, 2006.

[12] H. Ma and A. A. Girgis, "Identification and tracking of harmonic sources in a power system using a Kalman filter," IEEE Transactions on Power Delivery, vol. 11, no. 3, pp. 1659-1665, 1996.

[13] J. R. de Carvalho, C. A. Duque, M. V. Ribeiro, A. S. Cerqueira, T. L. Baldwin, and P. F. Ribeiro, "A PLL-based multirate structure for time-varying power systems harmonic/interharmonic estimation," IEEE Transactions on Power Delivery, vol. 24, no. 4, pp. 1789-1800, 2009.

[14] M. Mojiri, M. Karimi-Ghartemani, and A. Bakhshai, "Processing of harmonics and interharmonics using an adaptive notch filter," IEEE Transactions on Power Delivery, vol. 25, no. 2, pp. 534-542, 2010.

[15] A. Özdemir, İ. Yazıcı, and C. Vural, "Fast and robust softwarebased digital phase-locked loop for power electronics applications," IET Generation, Transmission and Distribution2013, vol. 12, no. 7, pp. 1435-1441.

[16] A. Ozdemir, "A digital adaptive filter for detecting harmonic, active and reactive currents," Measurement Science and Technology, vol. 15, no. 7, pp. 1316-1322, 2004.

[17] "Testing and measurement techniques: general guide on harmonics and interharmonics measurements and instrumentation, for power supply systems and equipment connected thereto," IEC 61000-4-7, 2005.

[18] O. Salor, "Spectral correction based method for interharmonics analysis of power signals with fundamental frequency deviation," Electric Power Systems Research, vol. 79, no. 7, pp. 10251031, 2009. 


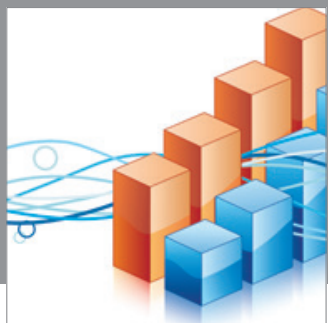

Advances in

Operations Research

mansans

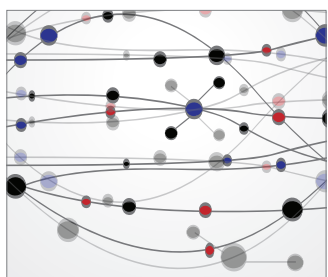

The Scientific World Journal
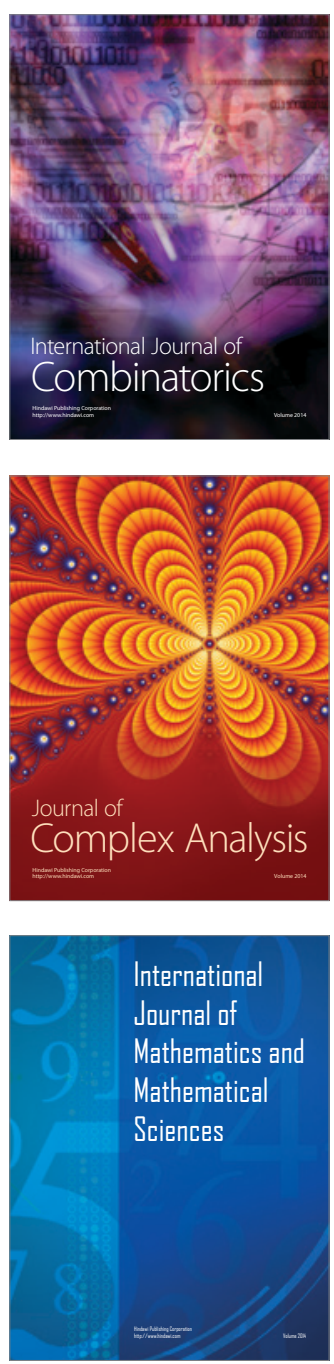
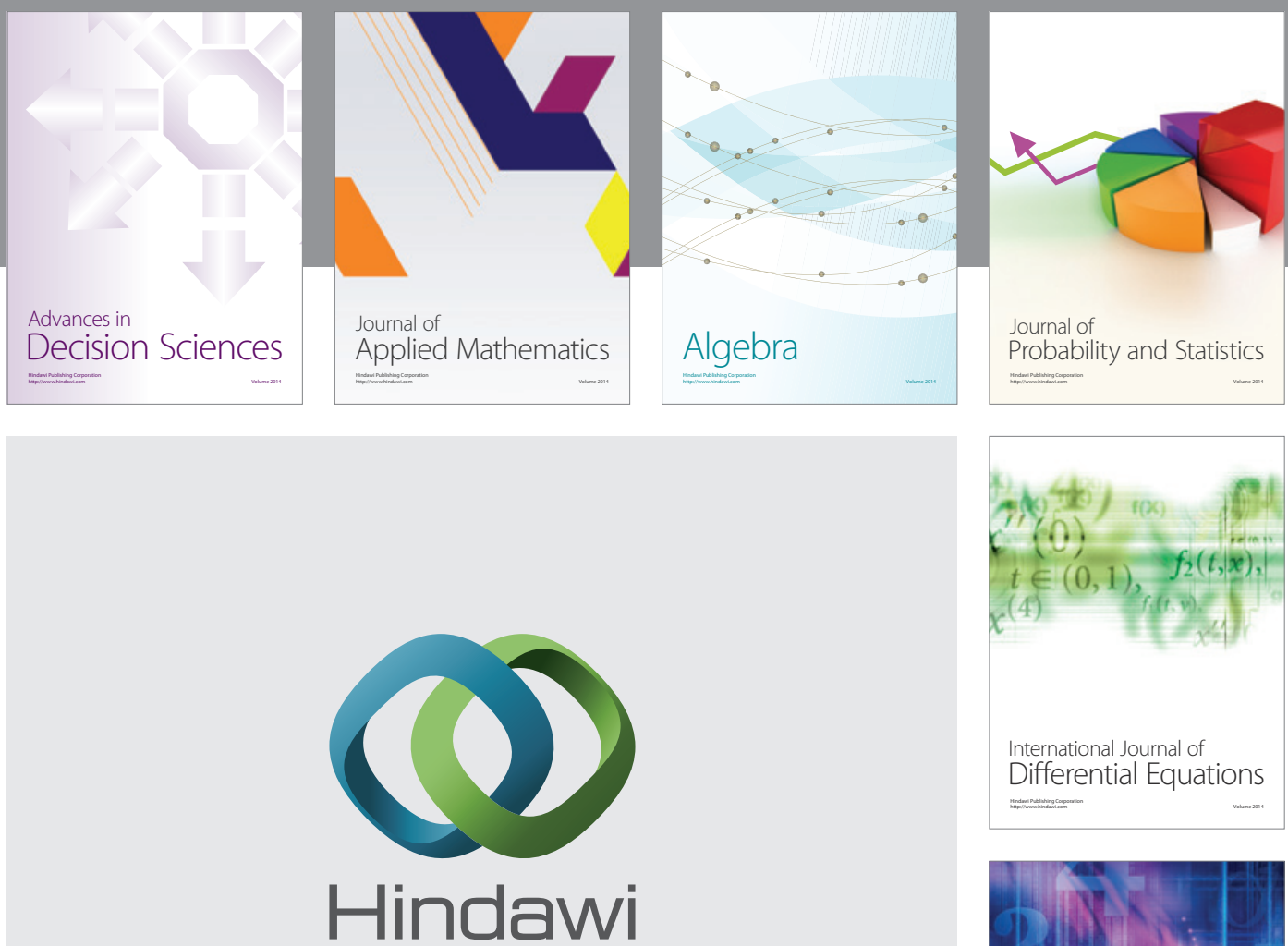

Submit your manuscripts at http://www.hindawi.com
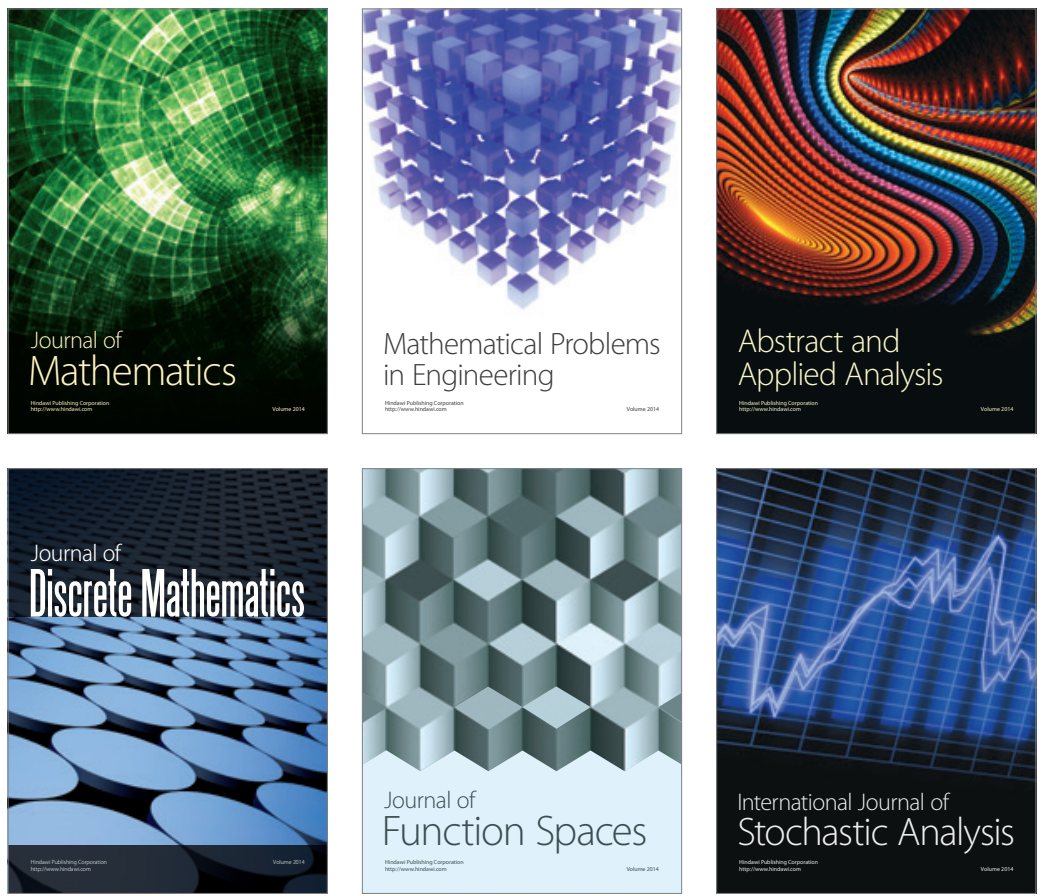

Journal of

Function Spaces

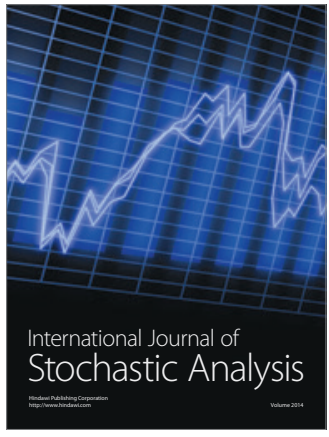

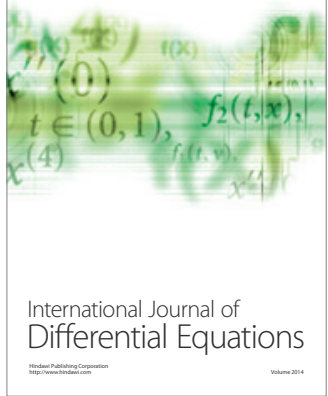
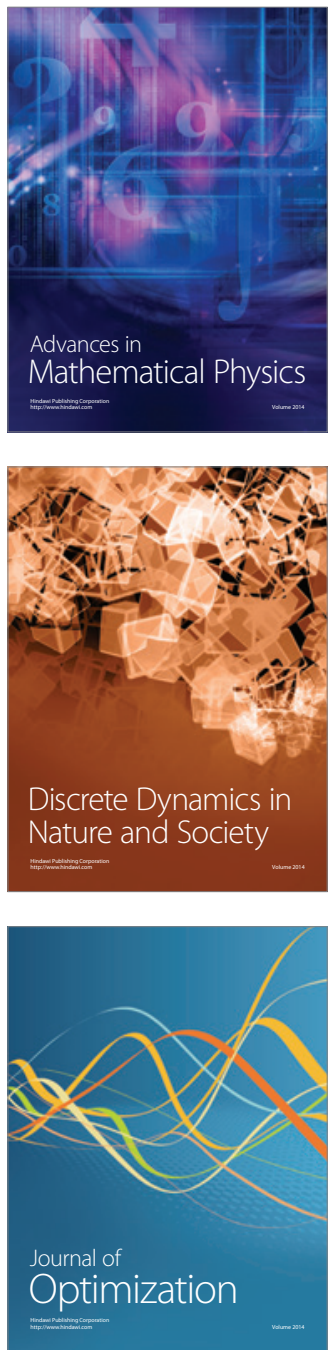